\title{
Upper gastrointestinal bleeding: risk factors for mortality in two urban centers in Latin America
}

\author{
C. H. Morales-Uribe' ${ }^{1}$ S. Sierra-Sierra ${ }^{2}$, A. M. Hernández-Hernández², A. F. Arango-Durango² and \\ G. A. Lopez ${ }^{2}$
}

${ }^{\text {} S e r v i c i o ~ d e ~ C i r u g i ́ a . ~ F a c u l t a d ~ d e ~ M e d i c i n a ~ U n i v e r s i d a d ~ d e ~ A n t i o q u i a . ~ H o s p i t a l ~ U n i v e r s i t a r i o ~ S a n ~ V i c e n t e ~ d e ~ P a u ́ l ~}$ (Medellín-Colombia). ${ }^{2}$ Universidad CES. Colombia

\begin{abstract}
Objective: To describe the experience with upper gastrointestinal bleeding (UGIB) in two major Latin American hospitals, its main causes, treatment and prognosis, while exploring some risk factors associated with death.

Design: Prospective cohort study.

Patients and Methods: We included 464 patients older than 15 years of age from two reference centers. We studied some demographic variables, history, clinical presentation, treatment and mortality. We explored the association between those variables and death.

Results: The mean age was 57.9 years, and the male: female ratio was $1.4: 1$. Three hundred and fifty-nine patients $(77.4 \%)$ were seen for gastrointestinal bleeding (outpatient bleeding) and 105 patients $(22.6 \%)$ were inpatients seen for UGIB. A total of $71.6 \%$ of patients admitted with the diagnosis of upper GI bleeding underwent upper GI endoscopy (EGD) within 24 hours. The main causes of bleeding were peptic ulcer (190 patients, 40.9\%), erosive disease (162 patients, 34.9\%) and variceal bleeding (47 patients, 10.1\%). Forty-four patients died (9.5\%). Patients who presented with bleeding due to other causes during hospitalization had a higher mortality risk than those whose complaints were related to gastrointestinal bleeding (RR 2.4, 95\% CI 1.2-4.6). An increasing number of comorbidities, such as those described in the Rockall score, were also associated with a higher risk of mortality (RR 2.5, 95\% CI 1.1-5.4).

Conclusion: Intrahospital upper GI bleeding and the presence of comorbidities are risk factors for a fatal outcome. Identifying patients with a higher risk would help improve the management of patients with UGIB.
\end{abstract}

Key words: upper gastrointestinal bleeding, risk factors, mortality.

C. H. Morales Uribe, S. Sierra Sierra, A. M. Hernández Hernández, A. F. Arango Durango, G. A. López. Upper gastrointestinal bleeding: risk factors for mortality in two urban centers in latin america. Rev Esp Enferm Dig 2011; 103: 20-24.

Received: 25-03-10.

Accepted: 25-05-10.

Correspondence: C. H. Morales Uribe. Hospital Universitario San Vicente de Paúl (Medellín-Colombia).cmorales@medicina.udea.edu.co

\section{INTRODUCTION}

Upper gastrointestinal bleeding is a common medical emergency and a frequent cause of morbidity and mortality. The mortality rates published in recent decades range from 8 to $14 \%$. (1-4) UGIB, which is defined as a bleeding above the ligament of Treitz, is five times more common than lower gastrointestinal bleeding (LGIB) and is more frequent in older male patients. Peptic ulcers have been recognized as the leading cause of UGIB $(2,5,6)$, although recent studies have shown a decrease in the percentage $(7,8)$. The use of proton pump inhibitors (PPIs) and the eradication of the Helicobacter pylori has decreased in recent decades, as has the percentage of patients who present with a reoccurrence of bleeding; however, the mortality rate has remained stable despite these and other developments, such as endoscopic treatment $(9,10)$, probably due to the increase in the average age of patients and the frequent and continuous use of nonstero idal anti-inflammatory drugs (NSAIDs) $(11,12)$.

The literature has reported numerous prognostic factors associated with death due to upper gastrointestinal bleeding (UGIB). $(11,12)$ The objective of this study was to present the experience with UGIB from two reference center hospitals of a Latin American country and its main causes, treatment and prognosis and to explore some risk factors associated with mortality.

\section{PATIENTS AND METHODS}

We conducted a prospective cohort study with 464 patients; 404 were selected from the San Vicente de Paúl de Medellín University Hospital (Colombia) from April 18, 2007 to August 31, 2008, and 60 patients were selected from the Medellín General Hospital from May 27, 2008 and August 28, 2008, both of which are reference centers in the city of Medellín (Colombia). 
The inclusion criteria included patients who were older than 15 years of age, who were admitted to a hospital with a diagnosis of UGIB or developed UGIB during hospitalization, and who underwent upper gastrointestinal endoscopy (EGD). To gather information, we used a form that included the variables of age, gender, period between admission and the conduction of endoscopy, hemodynamic status at admission, history of gastrointestinal bleeding, clinical presentation, comorbidities, use of a nasogastric tube, endoscopic diagnosis, duration of hospitalization, treatment and mortality.

The hemodynamic status was defined as unstable if the patient had a systolic blood pressure below $90 \mathrm{mmHg}$. The use of a nasogastric tube referred to its use at admission to confirm the presence of an upper acute bleeding or as part of UGIB management.

Hospital bleeding referred to the upper gastrointestinal bleeding that occurred in patients who were hospitalized for causes other than GI bleeding and who presented with bleeding during hospitalization.

For analysis of the data, we used the statistical program SPSS 16.0. We conducted a univariate analysis to explore the behavior of the variables, the quality of the data and the presence of external values. This analysis used descriptive statistics, such as means, proportions, standard deviations and ranges. We also conducted a bivariate analysis to explore the associations between some independent variables and the main outcome mortality. This was performed using the chi-square statistic and the Mann Whitney U test.

The protocol was approved by the ethics committee of the San Vicente de Paúl University Hospital and the Medellín General Hospital.

\section{RESULTS}

The general characteristics of the patients are shown in Table I.

Table I. General characteristics

\begin{tabular}{lc}
\hline Characteristic & $n(\%)$ \\
\hline Age & \\
$<60$ & $231(49,8)$ \\
$60-79$ & $161(34,7)$ \\
$80+$ & $72(15,5)$ \\
Total & $464(100)$ \\
Gender & \\
Male & $273(58,8)$ \\
Female & $191(41,2)$ \\
Reason for consultation & \\
Hematemesis & $240(66,9)$ \\
Manes & $217(60,4)$ \\
Hematochezia & $59(16,4)$ \\
Syncope & $39(10,9)$ \\
Other & $91(25,3)$ \\
Previous Episodes & $108(23,3)$ \\
\hline
\end{tabular}

The average age was 57.9 years (DE 20). The youngest patient was 15 years old, and the oldest was 93 years old. The male: female proportion was 1.4:1. The most frequent reason for consultation was hematemesis $(66.9 \%)$. Sixty-four patients $(13.8 \%)$ were in shock at the time of bleeding, defined as a systolic blood pressure below $90 \mathrm{mmHg}$.

One hundred and eight patients (23.3\%) had a history of gastrointestinal bleeding.

Some comorbidities and history factors were smoking $35.8 \%$, alcohol intake $27.8 \%$, consumption of NSAIDs $15.9 \%$, dyspepsia $15.9 \%$, diabetes mellitus $10.8 \%$, cirrhosis $8.6 \%$, use of anticoagulants $5.8 \%$, portal hypertension $5.4 \%$ and chronic hepatitis $1.7 \%$.

Three hundred and nine patients $(77.3 \%)$ had outpatient gastrointestinal bleeding (extra-hospital) and 105 patients $(22.6 \%)$ presented with bleeding while hospitalized (intra-hospital). We used a nasogastric tube in $5 \%$ of the patients.

We conducted an EGD within 24 hours in $71.6 \%$ and within two days in $84.4 \%$ of the patients who were admitted for UGIB.

Peptic ulcers were the main cause of bleeding (41\%) in the studied population, and the most common were gastric ulcers. Erosive disease was responsible for $34.9 \%$ of the bleedings, followed by variceal bleeding (10.1\%). Table II shows these and other results.

Table II. Causes of upper gastrointestinal bleeding

\begin{tabular}{lc}
\hline Causes of bleeding & $n(\%)$ \\
\hline Peptic Ulcer & $190(41 \%)$ \\
Gastric & $107(23,1 \%)$ \\
Duodenal & $70(15,1 \%)$ \\
Esophageal & $13(2,8 \%)$ \\
Erosive Disease & $162(34,9 \%)$ \\
Gastritis & $60(12,9 \%)$ \\
Esophagitis & $63(13,6 \%)$ \\
Duodenitis & $39(8,4 \%)$ \\
Variceal Disease & $47(10,1 \%)$ \\
Esophageal & $39(8,4 \%)$ \\
Gastric & $8(1,7 \%)$ \\
Hypertensive Gastropathy & $20(4,3 \%)$ \\
Mallory Weiss Syndrome & $18(3,9 \%)$ \\
Malignant Lesions & $14(3,0 \%)$ \\
Gastric & $6(1,3 \%)$ \\
Dieulafoy's Lesion &
\end{tabular}

About $77 \%$ of the peptic ulcers were classified according to Forrest; the most frequent degree was Forrest III $(38.4 \%)$. The ulcers that were classified as Forrest I and Forrest II comprised $26 \%$ of the cases.

The pharmacological treatment received by the patients had the following distribution: $344(74.1 \%)$ received parenteral proton pump inhibitors, 244 (52.6\%) 
received $\mathrm{H} 2$ blockers, 33 (7.1\%) received beta blockers and octreotide was administered with less frequency ( 25 (5.4\%) of the patients), while vasopressin and somatostatin were administered to one patient each $(0.2 \%)$.

Sixty-two (15.5\%) patients with Forrest Ia, Ib and IIa bleeding required endoscopic treatment for variceal disease. Those patients were injected with adrenaline alone. We performed band ligation in $15(3.2 \%)$ of the patients with variceal disease with active bleeding or signs of recent bleeding. We also performed a new endoscopic evaluation in $79(17.4 \%)$ patients. This figure includes patients who underwent endoscopy for suspected reoccurrence of bleeding who were at risk for a reoccurrence of bleeding during the first endoscopy and cases that did not have a satisfactory first evaluation. From this group, eight patients (10.1\%) required a new endoscopic treatment, six with an ulcer disease and two with variceal disease.

We conducted an angiographic evaluation in three patients $(0.6 \%)$. In one patient, we observed a bleeding of the gastroduodenal artery, which we controlled with embolization. In another patient, we observed active bleeding from the left gastric artery; however, we were not able to control this with embolization, and it required a gastrectomy for gastric carcinoma. The arteriography of the third patient did not show any bleeding or vascular malformation, and the recovery was successful without further treatment. Only $10(2.2 \%)$ patients required surgical treatment. The diagnoses of these patients were gastric carcinoma (5 cases), gastric ulcer ( 1 case), duodenal ulcer ( 1 case), Dieulafoy's lesion (1 case), erosive gastritis ( 1 case), and anastomotic mouth ulcer (1 case). We were not able to control the bleeding of the patient with a duodenal ulcer in the first procedure, and immediate surgery was indicated. The Dieulafoy's lesion was not identified in the endoscopy and required surgical treatment. The reoccurrence of bleeding was also an indication for surgery. During the period of the study, another three patients were operated with urgency for ulcer disease without an endoscopy.

The patients who presented with bleeding while hospitalized for another reason remained hospitalized for 20.6 days, while those who were seen for outpatient UGIB had an average hospitalization of 7 days $(\mathrm{p}<0.05)$.

Forty-four (9.5\%) patients died. Tables III and IV show the distribution of the patients according to their pre- and post-endoscopy Rockall score. The mortality according to the value and its comparison with the probability of death according to the Rockall scale are also shown in Tables III and IV.

Table V shows the exploratory bivariate analysis of some variables that could be related to the probability of death. Patients who bleed during hospitalization for a different cause had a higher risk of dying than those patients who were seen for UGIB (RR 2.4, 95\% CI 1.2-4.6). The rising value of the comorbidities assigned by Rockall was equally associated with the probability of death (RR 2.5, $95 \%$ CI 1.1-5.4).

\begin{tabular}{|c|c|c|c|}
\hline $\begin{array}{l}\text { Pre-endoscopy } \\
\text { score }\end{array}$ & $n(\%)$ & Death $n(\%)$ & $\begin{array}{l}\text { Rockall death } \\
\text { probability (\%) }\end{array}$ \\
\hline 0 & $158(34,1)$ & $9(5,7)$ & $0(0-12)$ \\
\hline 1 & $108(23,3)$ & $8(7,4)$ & $3(0,6-5)$ \\
\hline 2 & $82(17,7)$ & $9(11,0)$ & $6(3-9)$ \\
\hline 3 & $71(15,3)$ & $11(15,5)$ & $12(9-16)$ \\
\hline 4 & $31(6,7)$ & $4(12,9)$ & $21(17-25)$ \\
\hline 5 & $11(2,4)$ & $2(18,2)$ & $35(27-43)$ \\
\hline 6 & $3(0,6)$ & $1(33,3)$ & $62(50-73)$ \\
\hline Total & 464 & $44(9,5)$ & \\
\hline
\end{tabular}

\begin{tabular}{|c|c|c|c|}
\hline $\begin{array}{c}\text { Post-endoscopy } \\
\text { score }\end{array}$ & $n(\%)$ & Death $n(\%)$ & $\begin{array}{c}\text { Rockall death } \\
\text { probability }\end{array}$ \\
\hline 0 & $49(10,6)$ & $3(6,1)$ & $0(0-1)$ \\
\hline 1 & $111(23,9)$ & $5(4,5)$ & $0(0-1)$ \\
\hline 2 & $86(18,5)$ & $7(8,1)$ & $0(0-1)$ \\
\hline 3 & $80(17,2)$ & $7(8,8)$ & $3(0-4)$ \\
\hline 4 & $76(16,4)$ & $14(18,4)$ & $8(4-12)$ \\
\hline 5 & $32(6,9)$ & $4(12,5)$ & $11(6-15)$ \\
\hline 6 & $19(4,1)$ & $1(5,3)$ & $12(6-17)$ \\
\hline 7 & $9(1,9)$ & $2(22,2)$ & $23(15-31)$ \\
\hline 8 & $2(0,4)$ & $1(50,0)$ & $40(30-51)$ \\
\hline Total & $464(100)$ & $44(9,5)$ & \\
\hline
\end{tabular}

\section{DISCUSSION}

The general characteristics of the studied group, including age, gender, history of previous bleeding, clinical presentation and comorbidities, were similar to literature reports (13-16). The number of patients older than 60 years corresponded to half of the group; this percentage has increased according to recent studies $(13,15)$. The percentage of patients who took NSAIDs was lower than in other studies $(13,14)$.

A little more than $70 \%$ of the patients underwent EGD within the first 24 hours of admission. Current protocols suggest that an early risk stratification of patients according to clinical and endoscopic criteria, and the practice of early endoscopy (before 24 hours), allow for a prompt and reliable release of those patients with a low risk and improve the prognosis of high-risk patients. (17-19) Despite having no significant impact on mortality, studies have shown a reduction in the resources used, number of transfusions, duration of hospitalization, reoccurrence of bleeding and the need for emergency surgery when an early endoscopy is performed; in addition, early endoscopy does not pose a risk to the patient $(17,20)$. In their recent study, Gómez et 
Table V. Bivariate analysis between some variables and death

\begin{tabular}{|c|c|c|c|c|}
\hline \multicolumn{2}{|c|}{ Variable } & \multirow{2}{*}{$\begin{array}{c}\begin{array}{c}\text { Death } n(\%) \\
44(9,5 \%)\end{array} \\
26(9,5) \\
18(9,4)\end{array}$} & \multirow{2}{*}{$\frac{R R(I C 95 \%)}{1,0(0,5-1,9)}$} & \multirow{2}{*}{$\frac{\text { Value } p}{0,971}$} \\
\hline Gender & $\begin{array}{l}\text { Male } \\
\text { Female }\end{array}$ & & & \\
\hline $\begin{array}{l}\text { Previous } \\
\text { Episodes }\end{array}$ & $\begin{array}{l}\text { Yes } \\
\text { No }\end{array}$ & $\begin{array}{c}8(7,4) \\
36(10,1)\end{array}$ & $0,7(0,3-1,6)$ & 0,401 \\
\hline $\begin{array}{l}\text { Bleeding } \\
\text { site }\end{array}$ & $\begin{array}{l}\text { Inpatient } \\
\text { Outpatient }\end{array}$ & $\begin{array}{c}17(16,2) \\
27(7,5)\end{array}$ & $2,4(1,2-4,6)$ & 0,008 \\
\hline $\begin{array}{r}\text { You } \\
\text { Fo } \\
8\end{array}$ & $\begin{array}{l}\text { ounger than } 60 \\
\text { Form } 60 \text { to } 79 \\
80 \text { and older }\end{array}$ & $\begin{array}{c}19(8,2) \\
15(9,3) \\
10(13,9)\end{array}$ & $\begin{array}{l}1,6(0,5-2,5) \\
1,8(0,7-4,3)\end{array}$ & 0,189 \\
\hline Shock & $\begin{array}{l}\text { Yes } \\
\text { No }\end{array}$ & $\begin{array}{c}34(15,6) \\
10(8,5)\end{array}$ & $0,5(0,2-1,1)$ & 0,071 \\
\hline Comorbidities* & $\begin{array}{l}0 \\
2 \\
3\end{array}$ & $\begin{array}{c}32(8,6) \\
0(0) \\
12(19,0)\end{array}$ & $\begin{array}{c}\mathrm{NA} \\
2,5(1,1-5,4)\end{array}$ & 0,038 \\
\hline Bleeding stigmata & $\begin{array}{l}0 \\
2\end{array}$ & $\begin{array}{c}37(9,2) \\
7(11,1 \%)\end{array}$ & $0,8(0,3-1,9)$ & 0,635 \\
\hline Diagnosis* & $\begin{array}{l}0 \\
1 \\
2\end{array}$ & $\begin{array}{c}7(6,6) \\
35(10,2) \\
2(14,3)\end{array}$ & $\begin{array}{c}1,6(0,7-4,1) \\
2,4(0,3-14,9)\end{array}$ & 0,208 \\
\hline
\end{tabular}

Chi tendency

Values of comorbidities according to the Rockall score:

0 No major comorbidity.

2 Heart failure, ischemic heart disease or other illness.

3 Renal failure, liver failure or carcinomatosis.

Value of stigmata of recent bleeding according to the Rockall score:

0 None, dark spot.

2 Blood in the gastrointestinal tract, adherent clot, visible vessel, bleeding vessel.

Diagnostic value according to the Rockall score:

0 No lesion, Mallory Weiss

1 Other diagnoses

2 Upper gastrointestinal cancer

al. describe a scale based on clinical and laboratory parameters, which allows for the identification of patients who really need an urgent endoscopy(21).

Peptic ulcer disease has been recognized as the leading cause of UGIB $(1,3,7,14,15)$. The classic study of Rockall, with more than 4.000 patients, stated that peptic ulcer disease was responsible for $46 \%$ of the upper GI bleeds (53\% duodenal, 35\% gastric, $8 \%$ esophageal and 4\% mixed). (5) In this study, peptic ulcer was the main cause of bleeding (41\%), among which gastric ulcers were the most common source. However, some question whether the incidence of ulcers is decreasing, or perhaps less published $(7,8)$. Boonpongmanee et al. (7) reported data that support this affirmation, with an incidence of peptic ulcers of only $20.6 \%$ to $31.8 \%$. Czernichow et al. reported similar values (8).

The majority of peptic ulcers $(42 \%)$ corresponded to the Forrest III classification, followed in descending order by class IIC (20\%), IIB (17\%), IIA (17\%) and I (18\%).
This may reflect the widespread and protocol-based use of infusions of proton pump inhibitors beginning when the patient is admitted.

Although the pharmacological approach is the cornerstone of treatment, interventional endoscopy is an excellent complement in patients who continue to have active bleeding, and a few cases require interventional radiology or even surgery. In the studied group, the interventional endoscopy therapy was unimodal-injection epinephrine. The efficacy of this treatment is suboptimal and must be used in combination with other methods (22-24).

The current trend is to conduct a second endoscopy only in high-risk patients (clinical or endoscopic), those in whom the first EGD was technically difficult or impossible and those with a reoccurrence of bleeding, which represented $17.4 \%$ of the patients in this study (18).

Recent literature shows a decrease in the need for surgical management for UGIB (needed in about $4-10 \%$ of the patients) $(2,14,15,19)$; in this report, only $2.2 \%$ of the patients required surgical treatment, and this may reflect early consultation, timely attention given to the patients, management based on proton pump inhibitors, early endoscopy and the possibility of an interventional radiological treatment with embolization by selective catheterization (25).

There was a significant difference between the observed deaths and the probability of death in patients with a pre-endoscopic Rockall score greater than or equal to four and with a post-endoscopic score greater than or equal to six. This is probably due to the small number of patients with these values. For the lower values, the Rockall score offers a good predictive capacity in this group.

The mortality rate of this group is not different from other publications $(1-4,26)$.

The literature presents multiple risk factors associated with death, and some authors have suggested scores to classify the risk of patients with UGIB. In the exploration of risk in this group, we found two variables associated with death: the occurrence of bleeding in the hospital (RR 2.4, 95\% CI 1.2-4.6) and the presence of comorbidities (RR 2.5, 95\% CI 1.1-5.4), defined according to Rockall (5). Klebl et al. reported similar findings, concluding that mortality in patients with intrahospital bleeding is due not to one or a few but to several comorbidities, which are generally severe (27). A second article from the same authors mentions some of these comorbidities (28).

These findings should prompt the identification of patients who present with a higher risk of developing a fatal outcome; this will contribute to the improvement of the management of patients with UGIB, including an early therapeutic intervention.

\section{REFERENCES}

1. Palmer K. Acute upper gastrointestinal haemorrhage. Br Med Bull 2007; 83: 307-24.

2. Van Leerdam ME, Vreeburg EM, Rauws EAJ, Geraedts AAM, Tijssen JGP, Reitsma JB, et al. Acute Upper GI Bleeding: Did Anything Change?, Time Trend Analysis of Incidence and Outcome of Acute 
Upper GI Bleeding Between 1993/1994 and 2000. Am J Gastroenterol 2003; 98: 1494-9.

3. Esrailian E, Gralnek IM. Nonvariceal Upper Gastrointestinal Bleeding: Epidemiology and Diagnosis. Gastroenterol Clin N Am 2005; 34: $589-605$

4. Tariq SH, Mekhjian G. Gastrointestinal Bleeding in Older Adults Clin Geriatr Med 2007; 23: 769-84.

5. Rockall TA, Logan RFA, Devlin HB. Incidence of and mortality from acute upper Gastrointestinal haemorrhage in the United Kingdom. BMJ 1995; 311: 222-6.

6. Gralnek IM, Barkun AN, Bardou M. Management of Acute Bleeding from a Peptic Ulcer. N Engl J Med 2008; 359: 928-37.

7. Boonpongmanee S, Fleischer DE, Pezzullo JC, Collier K, Mayoral $\mathrm{W}, \mathrm{Al}-\mathrm{Kawas} \mathrm{F}$, et al. The frequency of peptic ulcer as a cause of upper-GI bleeding is exaggerated. Gastrointest Endosc 2004; 59: 78894.

8. Czernichow P, Hochain P, Nousbaum JB, Raymond JM, Rudelli A, Dupas JL, et al. Epidemiology and course of acute upper gastro-intestinal haemorrhage in four French geographical areas. Eur J Gastroenterol Hepatol 2000; 12: 175-81.

9. Barkun A, Sabbah S, Enns R, Armstrong D, Gregor J, Fedorak RNN, et al RUGBE investigators. The Canadian Registry on Nonvariceal Upper Gastrointestinal Bleeding and Endoscopy (RUGBE): Endoscopic Hemostasis and Proton Pump Inhibition are Associated with Improved Outcomes in a Real-Life Setting. Am Coll of Gastroenterology 2004; 1238-46.

10. Lewis JD, Bilker WB, Brensinger C, Farrar JT, Strom BL. Hospitalization and Mortality Rates From Peptic Ulcer Disease and GI Bleeding in the 1990s: Relationship to Sales of Nonsteroidal Anti-Inflammatory Drugs and Acid Suppression Medications. Am J Gastroenterol 2002; 97: 2540-9.

11. Theocharis GJ, Arvaniti V, Assimakopoulos SF, Thomopoulos KC, Xourgias V, Mylonakou I, Nikolopoulou VN. Acute upper gastrointestinal bleeding in octogenarians: Clinical outcome and factors related to mortality. World J Gastroenterol 2008 7; 14: 4047-53.

12. Lanas A, Perez-Aisa MA, Feu F, Ponce J, Saperas E, Santolaria S, Rodrigo L, Balanzo J, Bajador E, et al. A Nationwide Study of Mortality Associated with Hospital Admission Due to Severe Gastrointestinal Events and Those Associated with Nonsteroidal Antiinflammatory Drug Use. Am J Gastroenterol 2005; 100: 1685-93.

13. Jurado AM, Galván JT, Ruiz-Cabello Jiménez M, Pinel Julián LM. Evolution in the epidemiology of non-variceal upper digestive hemorrhage from 1985 to 2006. Rev Esp Enferm Dig 2008; 100: 273-7.

14. Di Fiore F, Lecleire S, Merle V, Hervé S, Christian Duhamel, Dupas $\mathrm{JL}$, et al. Changes in characteristics and outcome of acute upper gastrointestinal haemorrhage: a comparison of epidemiology and practices between 1996 and 2000 in a multicentre French study. Eur J Gastroenterol \& Hepatol 2005, 17: 641-7.
15. Thomopoulos KC, Vagenas KA, Vagianos CE, Margaritis VG, Blikas AP, Katsakoulis EC, et al. Changes in aetiology and clinical outcome of acute upper gastrointestinal bleeding during the last 15 years. Eur J Gastroenterol Hepatol 2004; 16: 177-82.

16. Gulmez SE, Lassen AT, Aalykke C, Dall M, Andries A, Andersen BS, Janse JM, Andersen M, Hallas J. Does statin protect against upper gastrointestinal bleeding? Br J Clin Pharmacol. 2009; 67: 460-5.

17. Spiegel BM, Vakil NB, Ofman JJ. Endoscopy for Acute Nonvariceal Upper Gastrointestinal Tract Hemorrhage: Is Sooner Better? Arch Intern Med 2001; 161: 1393-404.

18. Barkun A, Bardou M, Marshall JK.). Consensus Recommendations for Managing Patients with Nonvariceal Upper Gastrointestinal Bleeding. Ann Intern Med 2003; 139: 843-57.

19. Rockall TA, Logan RF, Devlin HB, Northfield TC. Influencing the practice and outcome in acute upper gastrointestinal haemorrhage. BMJ 1997; 41: 606-611.

20. Lim CH, Vani D, Shah SG, Everett SM, Rembacken BJ. The Outcome of Suspected Upper Gastrointestinal Bleeding With 24-Hour Access to Upper Gastrointestinal Endoscopy: A Prospective Cohort Study. Endoscopy 2006. 38: 581-5.

21. Gómez, MA; Pineda, LF; Ibañez, M; Otero, William; Arbeláez, V. Escala UNAL de predicción para identificar pacientes con hemorragia digestiva alta que necesitan endoscopia urgente. Rev Colomb Gastroenterol. 2006; 21: 244-58.

22. Barkun AN, Bardou M, Kuipers EJ, MD; Sung J, Hunt RH, Martel $\mathrm{M}$, Sinclair $\mathrm{P}$, the International Consensus Upper Gastrointestinal Bleeding International Consensus Recommendations on the Management of Patients With Nonvariceal Upper Gastrointestinal Bleeding Conference Group. Ann Intern Med. 2010; 152: 101-13.

23. Application of endoscopic hemoclips for nonvariceal bleeding in the upper gastrointestinal tract. Guo SB, Gong AX, Leng J, Ma J, Ge LM. World J Gastroenterol 2009 r 14; 15: 4322-6.

24. Ding YJ, ZhaoL, Liu J, Luo HS Clinical and endoscopic analysis of gastric Dieulafoy's lesion. World J Gastroenterol 2010; 16: 631-5.

25. Loffroy R, Guiu B Role of transcatheter arterial embolization for massive bleeding from gastroduodenal ulcers. World J Gastroenterol 2009; 15: 5889-97.

26. Manguso F, Riccio E, Bennato R, Picascia S, Martino R, De Nucci G, Fiorito R, Balzano A. In-hospital mortality in non-variceal upper gastrointestinal bleeding Forrest 1 Patients. Scand J Gastroenterol 2008. 4: 1-10.

27. Klebl F, Bregenzer N, Schofer L, Tamme W, Langgartner J, Scholmerich J, Messmann H. Comparison of inpatient and outpatient upper gastrointestinal haemorrhage. Int Colorectal Dis 2005. 20: 36875.

28. Klebl F, Bregenzer N, Schofer L, Tamme W, Langgartner J, Scholmerich J, Messmann H. Risk factors for mortality in severe upper gastrointestinal bleeding. Int J Colorectal Dis 2005. 20: 49-56. 